\title{
UNIQUE EQUILIBRIA AND SUBSTITUTION EFFECTS IN A STOCHASTIC MODEL OF THE MARRIAGE MARKET
}

\author{
COLIN DECKER, ELLIOTT H. LIEB, ROBERT J. MCCANN, AND BENJAMIN K. STEPHENS
}

\begin{abstract}
Choo-Siow (2006) proposed a model for the marriage market which allows for random identically distributed noise in the preferences of each of the participants. The randomness is McFadden-type, which permits an explicit resolution of the equilibrium preference probabilities.

The purpose of this note is to prove uniqueness of the resulting equilibrium marriage distribution, and find a representation of it in closed form. This allows us to derive smooth dependence of this distribution on exogenous preference and population parameters, and establish sign, symmetry, and size of the various substitution effects, facilitating comparative statics. For example, we show that an increase in the population of men of any given type in this model leads to an increase in single men of each type, and a decrease in single women of each type. We show that an increase in the number of men of a given type increases the equilibrium transfer paid by such men to their spouses, and also increases the percentage of men of that type who choose to remain unmarried. While the above trends may not seem surprising, the verification of such properties helps to substantiate the validity of the model. Moreover, we make unexpected predictions which could be tested: namely, the percentage change of type $i$ unmarrieds with respect to fluctuations in the total number of type $j$ men or women turns out to form a symmetric positive-definite matrix $r_{i j}=r_{j i}$ in this model, and thus to satisfy bounds such as $\left|r_{i j}\right| \leq\left(r_{i i} r_{j j}\right)^{1 / 2}$.

Along the way, we give a new proof for the existence of an equilibrium, based on a strictly convex variational principle and a simple estimate, rather than a fixed point theorem. Fixed point approaches to the existence part of our result have been explored by others [6] 8] [12, but are much more complicated and yield neither uniqueness, nor comparative statics, nor an explicit representation of the solution.
\end{abstract}

JEL Classification: J12, C62, C78, C81, D03, Z13

KEYWORDS: Choo-Siow, marriage market, matching, random, unique equilibrium, comparative statics, convex analysis.

Date: March 27, 2018.

The authors are grateful to Aloysius Siow for attracting their attention to this question, and for many fruitful discussions. This project developed in part from the Master's research of CD; however, the present manuscript is based a new approach which greatly extends (and largely subsumes) the results of his thesis 9], and of the 2009 preprint by three of us entitled When do systematic gains uniquely determine the number of marriages between different types in the Choo-Siow matching model? Sufficient conditions for a unique equilibrium. CD, RJM and BKS are pleased to acknowledge the support of Natural Sciences and Engineering Research Council of Canada (NSERC) grant 217006-03 RGPIN; CD also benefitted from an Undergraduate Student Research Award (URSA) held in the summer of 2009. EHL acknowledges a United States National Science Foundation grant PHY-0965859. (C)2011 by the authors. 


\section{INTRODUCTION}

The classic transferable utility framework which Becker used to model the marriage market was augmented by Choo and Siow [7] to allow for the possibility that agents' preferences might be only partly determined by observable characteristics, and might therefore include a stochastic component depending on unobservable characteristics. The randomness was chosen to be McFadden type [19], and spreads the preferences of agents on one side of the marriage market over the entire type-distribution of agents on the other, thus yielding non-assortative matching, a feature of the marriage market that has long been observed empirically [7]. The model is non-parametric and highly tractable; given an observation of marriages between different types of agents, there is a simple closed form, 'point-identified' expression for the average utility (or 'total gains') generated by each type of marriage. Of use to econometricians, demographers, and economic theorists is a solution to the backwards problem: given the total gains to each type of marriage and population data, what can be said about possible distributions of marriages corresponding to those parameters? Basic questions include existence and uniqueness of such a marital distribution; computing comparative statics is even more interesting. How will the marital distribution respond to changes in composition of the population? How will it change due to policy shocks whose effects can be summarized by revising the total gains parameters? The purpose of the present note is to address these questions.

A priori, it is not evident whether each choice of gains parameters leads to the existence of a distribution of marriages which clears the market [6] [12] 8]. Although it is not the main point of our paper, we begin by reconfirming that it does. We then go on to show this equilibrium is unique, and give an explicit formula for the resulting marriage distribution, essentially solving the model completely. This allows us to derive smooth dependence of the resulting equilibrium state on the specified population and preference parameters, and establish sign, symmetry and bounds on the response of the predicted distribution of marriages to changes in each parameter. Although existence of an equilibrium was also discussed by Choo, Seitz and Siow [6] and Fox [12] (and by Dagsvik [8] for a related model), our proof relies on the reformulation of the problem as a variational minimization, hence is simpler than the fixed point argument they suggest. More importantly, it yields the solution in closed form. Our uniqueness result is the first concerning this model [5], and is based on convexity (in appropriate variables) of the new variational principle formulated in Section \$5. The variational technique we use is powerful, because it characterizes the endogenous variables of interest as the critical points of a function. Indeed, the competitive equilibrium in this model turns out to be realized as the minimum of a strictly convex function (given in Theorem 1 below), facilitating its subsequent analysis.

1.1. Organization. The remainder of this introduction provides further motivation and background for the Choo-Siow model. Section $\$ 2$ details the model and could be skipped by readers familiar with the Choo-Siow model, except that it ends with a summary placing our results in the context of related literature. Further comments concerning the derivation of the model may be found in Appendix A Section $\$ 3$ states our results formally; it is followed by a section containing derived statics and one further conjecture. Section \$5 
proves the existence and uniqueness of equilibria, while 96 is devoted to the comparative statics established in Theorem 2, Section 87 establishes the further comparative static assertions of Corollary 3 and \$4

1.2. Further remarks. The random component of agent preferences is a salient feature of the Choo-Siow model. Due to this randomness, the equilibrium marriage distribution predicted by the model will not be positive assortative, even when the observed attributes of the agents are one-dimensional. This is consistent with empirical data. Even in experiments where the agents are parameterized by ordered types, such as age, observed marital data will almost never be genuinely assortative. For example, in any given population it is unlikely to be true that the age of the youngest woman married to a 34 year old man always exceeds that of the oldest woman married to a 33 year old. Similarly, one always finds matches in large populations that pair high with low qualities as measured by any given ordered observable characteristic (e.g. income, years of education, etc.). Thus a strictly assortative framework fails to explain the presence of, for example, the existence of $\mathrm{PhD}$ graduates married to high-school drop-outs.

The classic transferable utility model of the marriage market, introduced by Gary Becker [1, in principle predicts how agents will marry given exogenous preference parameters. However, it has seldom been estimated. There are two main obstacles to estimating a model of the marriage market. First, equilibrium transfers in modern marriages (except in the case of dowries) are not observed. Hence any behavioural model that requires their presence in data is not identifiable. Second, real-world agents are described by discrete, multidimensional, possibly unordered, types. But the classic Becker model predicts positive assortative matching under the assumption that agent type is one dimensional, continuous, ordered, and that preferences are super-modular. This positive assortative matching is limiting, but does ensure that his model predicts a unique marital distribution.

The Choo-Siow model eliminates the structural assumptions of the classic model. First, it is not necessary to observe transfers in order to determine the equilibrium marriage distribution generated by the model. In fact, we provide an explicit formula for the equilibrium marriage distribution in terms of the derivative of the Legendre transform of a known function. Second, the model places no a priori structure on the nature or number of types that agents (men and women) can have. This allows consideration of a wide range of attributes, like race, religion, level of income, and educational achievements.

In this more realistic framework, with its lack of structure for the agents' deterministic preferences and types, the issue of whether there exists an equilibrium marital distribution, and if so whether it is unique, becomes a question of fundamental theoretical and econometric significance. The theoretical importance arises from the fact that uniqueness of equilibria in two-sided matching problems is usually not better than a generic property, except perhaps in certain convex programming settings like [10] [11, which include continuous Monge-Kantorovich matching [16] [4]. Further, the randomness considered in the model below is the commonly used extreme value logit type, thus any result that describes properties of the equilibrating matches has potentially wider applicability. The econometric importance arises from the fact that models of the marriage market are useful 
to econometricians only insofar as they make unique predictions of a marital distribution, given exogenous preferences. From a practical point of view having closed form solutions which permit comparative statics may be even more crucial.

\section{The Choo-Siow Marriage Matching Model}

Our presentation emphasizes the stochastic heterogeneity that differentiates the ChooSiow model from classical models. The competitive framework, which uses transfers of utility from spouses to equilibrate the market, is explored in detail in Choo-Siow [7] but treated here only in $\$ 7$. It should be noted at the outset that the methods developed here also apply to other non-transferable utility models present in the literature. For example, Dagsvik [8] develops a model of the marriage market which uses an assignment algorithm (deferred acceptance) rather than utility transfers to sort matches, but his equilibrium conditions are functionally similar to ours.

2.1. Setting. What is exogenous in this model are the observed types of men and of women, the numbers of men and women of each type in the population, and the total gains $\pi_{i j}$ of marriage between a man of observed type $i$ and a woman of observed type $j$, relative to both partners remaining single. The quantity $\pi_{i j}$ will not reappear until (17). On the other hand, individual agents have a utility function that depends on both an endogenous deterministic component that captures systematic utility, and an exogenous random one that models heterogeneity within the population of each given type. Thus the utility accrued by a man of type $i$ and specific identity $g$ who marries a woman of type $j$ is assumed to be:

$$
V_{i j g}^{m}=\eta_{i j}^{m}+\sigma \epsilon_{i j g}
$$

the case $j=0$ represents the utility of remaining single. The deterministic component is $\eta_{i j}^{m}$; its endogeneity can be interpreted to reflect the possibility of interspousal transfer, as in Choo-Siow [7] and 97 below. It is set in equilibrium, and depends explicitly on the type of the man and the type of the woman, and implicitly on market conditions, i.e. on the relative abundance or scarcity of men and women of each different type. The random term $\epsilon_{i j g}$ depends additionally on the specific identity of the man, but not on the specific identity of the woman. Hence a specific thirty-five year old man may have stronger than typical (with respect to his age group) attraction for fifty-year old women. But this attraction does not depend on whether, for example, the older woman has an especially strong attraction to younger men (assuming this latter characteristic is unobservable in the data and hence not reflected in $j$ ).

The random term is assumed to have the Gumbel extreme value distribution described in Appendix A. This distribution was introduced to the economics literature by McFadden [19. It represents a severe simplifying assumption in our model, but has recently relaxed in work by Galichon and Salanié [15] which allows heteroskedasticity, for example. Our homoskedastic model admits only a single scaling parameter $\sigma$ which measures the degree of randomness; its reciprocal can be interpreted as the signal to noise ratio. It is equal to unity in the original Choo-Siow model. For illustrative purposes, we will have occasion to 
allow $\sigma$ to vary and in doing so embed the Choo-Siow model in a one parameter family of models that differ by the degree of randomness present in them.

In contrast with deterministic matching models, agents of a particular type do not have a uniform preferred match. This is because their preferences depend on the random variable $\epsilon_{i j g}$. Using the Gumbel structure, the probability that a man of type $i$ prefers a woman of type $j$ among all other possible marital choices $k \in\{0, \ldots, J\}$ is given by

$$
\operatorname{Pr}(\text { Man of type }(i, g) \text { prefers a woman of type } j)=\frac{\exp \left(\frac{\eta_{i j}^{m}}{\sigma}\right)}{\sum_{k=0}^{J} \exp \left(\frac{\eta_{i k}^{m}}{\sigma}\right)}
$$

(see Appendix $\mathrm{A}$ for a derivation). This probability distribution is endogenous, because it depends on the various $\eta_{i k}^{m}$. Note that it does not depend on the specific identity $g$ of the man of type $i$, since the noise is identically distributed for each different $g$. Yet it is possible already to see how the equilibrium marriage output will differ markedly from a deterministic one. Whereas in the deterministic case all members of a given type typically have the same preferred match, here the preferred matches of type $i$ men are smeared across all female types according to the distribution defined by (21). The mean and spread of the smearing are determined by the endogenous values $\eta_{i j}^{m}$ and by $\sigma$, respectively.

Consider $\sigma \in[0, \infty]$. The case $\sigma=1$ corresponds to the Choo-Siow model where some smearing is present. The case $\sigma=0$ corresponds to a deterministic matching model, for which there is no smearing. Indeed, as $\sigma \rightarrow 0$, the largest exponentials dominate all others, and the probability that a man of type $i$ prefers a woman of type $j$ converges to 0 or $\frac{1}{\# \arg \max \left\{\eta_{i j}^{m} \mid 0 \leq j \leq J\right\}}$ depending on whether or not $\eta_{i j}^{m}$ weakly dominates all other preference parameters $\eta_{i k}^{m}$. Conversely, as $\sigma \rightarrow \infty$, the stochastic term dominates the utility function, and the resulting probability distribution converges to the uniform distribution. In this case, there is maximal smearing, as preferences are completely random, constrained only by availability of prospective partners to marry.

Female preferences are also smeared, and the equilibrium marriage distribution is determined when $\eta_{i j}^{m}$ and $\eta_{i j}^{f}$ are such that the number of desired marriages of each type is the same on both sides of the market.

We now elaborate on the Choo-Siow model. We henceforth fix $\sigma=1$; since preferences are relative, this normalization can always be attained by rescaling all of the preferences in the model.

2.2. The Choo-Siow model. Suppose we wish to predict the number of marriages between men and women of different types. The number of men of type $i$ is denoted $m_{i}$. The number of marriages of type $i$ men to type $j$ women is denoted $\mu_{i j}$. The number of type $i$ men (respectively type $j$ women) who choose to remain single is denoted by $\mu_{i 0}$ (and $\mu_{0 j}$ respectively). If each man marries his preferred woman, the equality

$$
\operatorname{Pr}(\text { Man of type }(i, g) \text { prefers a woman of type } j)=\frac{\mu_{i j}}{m_{i}}
$$


will be valid, or at least as the population size becomes large, the right hand side of the equality converges to the left hand side by the law of large numbers, or the maximum likelihood theorem.

Using equations (22)-(3) to compute the ratio of the probability that a man of type $i$ prefers a woman of type $j$ to the probability that he prefers to remain single, we arrive at the following formula:

$$
\mu_{i j}^{m}=\frac{e^{\eta_{i j}^{m}}}{e^{\eta_{i 0}^{m}}} \mu_{i 0}^{m}
$$

These $I \times J$ equations are in fact quasi-demand equations, because they indicate the number of type $\mu_{i j}$ marriages that men of type $i$ would like to participate in. Viewing the female market cohort as the supply side, there are analogous supply equations. Letting the utility acquired by a woman of type $j$ and specific identity $h$ who marries a man of type $i$ be

$$
V_{i j h}^{f}=\eta_{i j}^{f}+\epsilon_{i j h},
$$

the above analysis produces $I \times J$ supply equations of the form:

$$
\mu_{i j}^{f}=\frac{e^{\eta_{i j}^{f}}}{e^{\eta_{0 j}^{f}}} \mu_{0 j}^{f} .
$$

The equilibrium output in the Choo-Siow model is a specification of $\mu_{i j}$ for all $0 \leq i \leq I$, and all $0 \leq j \leq J$. This output is obtained by requiring that supply balance demand: $\mu_{i j}^{f}=\mu_{i j}^{m}$. Under this market-clearing hypothesis, we have the following equation. The endogenous parts of the $\eta_{i j}^{m}, \eta_{i j}^{f}, \eta_{i 0}^{m}, \eta_{0 j}^{f}$ are eliminated upon adding them to arrive at the definition of an exogenous 1 aggregated gains variable $\pi_{i j}$ associated to each observable type of marriage:

$$
\pi_{i j}:=\frac{\eta_{i j}^{m}+\eta_{i j}^{f}-\eta_{i 0}^{m}-\eta_{0 j}^{f}}{2}
$$

Using the market-clearing hypothesis, we may re-write the equilibrium condition $\mu_{i j}^{m}=$ $\mu_{i j}^{f}=: \mu_{i j}$ in terms of the exogenous variable $\pi_{i j}$ as follows:

$$
\frac{\mu_{i j}}{\sqrt{\mu_{i 0} \mu_{0 j}}}=e^{\pi_{i j}}
$$

Finally, letting $\Pi_{i j}=e^{\pi_{i j}}$, the equilibrium output is given by

$$
\frac{\mu_{i j}}{\sqrt{\mu_{i 0} \mu_{0 j}}}=\Pi_{i j}
$$

The equilibrium conditions expressed in equation (9) are implicit. They give necessary conditions for real numbers $\mu_{i j}$ to be an output of the Choo-Siow model. However, they are not sufficient; a secondary set of necessary conditions, population constraints, must

\footnotetext{
${ }^{1}$ See $₫ 7$ below or Choo and Siow [7] for an explanation in terms of spousal transfers.
} 
also be satisfied. Let there be $I$ types of men, and $J$ types of women. The number of men of type $i$ is denoted $m_{i}$, and the number of women of type $j$ is denoted $f_{j}$. The vector whose $i^{\text {th }}$ component is $m_{i}$, and whose $(I+j)^{t h}$ component is $f_{j}$, is denoted by $\nu$. Called the population vector, it has $(I+J)$ components and may also be denoted by $[m \mid f]$. A specification $\left(\mu_{i j}\right)_{\substack{0 \leq i \leq I \\ 0 \leq j \leq J}}$ of the number of marriages and singles of each type is called a marital distribution. The following population constraints must be satisfied by all marital distributions, and are a consequence of the definitions:

$$
\begin{gathered}
\mu_{i 0}+\sum_{j=1}^{J} \mu_{i j}=m_{i}, \\
\mu_{0 j}+\sum_{i=1}^{I} \mu_{i j}=f_{j}, \\
\mu_{i j} \geq 0 .
\end{gathered}
$$

Several questions naturally arise. We call these questions the Choo-Siow inverse problem:

Problem (Choo-Siow inverse problem) Given a gains matrix $\Pi=\left(\Pi_{i j}\right)$ and a population vector $\nu=[m \mid f]$, does there exist a unique marital arrangement generating $\Pi$ ? In other words, assuming the entries $\Pi_{i j}$ to be non-negative and $m_{i}$ and $f_{j}$ to be strictly positive, does exactly one matrix $\left(\mu_{i j}\right)$ with non-negative entries exis that satisfies (9)(12)? Furthermore, can the qualitative dependence of $\left(\mu_{i j}\right)$ on the exogenous parameters $\Pi=\left(\Pi_{i j}\right)$ and $\nu=\left[\nu_{i}\right]$ be described?

This problem is important for several reasons. First, the implicit conditions present in equation (9) are the equilibrium outcome of a competitive market. There are not so many realistic environments with finitely many agent types and many commodities which are known to generate unique competitive equilibria - except possibly generically. While there are generic uniqueness results for matching problems that can be reduced to convex programing problems such as Monge-Kantorovich matching, e.g. [16] [4] [10] [11, the stochastic heterogeneity prevents the equilibrium in our model from being formulated as such.

\footnotetext{
${ }^{2}$ Some readers have pointed out that for each realization of the randomness, balancing supply with demand amounts to solving a linear program. In this setting, existence of an equilibrium (and its generic uniqueness) are well-known [16 4]. However, instead of balancing supply with demand for each realization, the Choo-Siow model is based on balancing expected supply (4) with expected demand (6). Since the expected supply (or demand) need not correspond to the actual supply (or demand) for any realization of the randomness, existence of an equilibrium in the sense prescribed by Choo and Siow is not obvious. Unlike a Nash equilibrium, in which no individuals have both the incentive and the opportunity to change their marital status, in a Choo-Siow equilibrium this will only be true in some average sense. Never the less, the notion has proved useful empirically, and might even be construed to reflect the metastability (as opposed to stability) displayed by actual marriage markets. As simple examples show, such equilibria generally correspond to interior points in the feasible polytope defined by the population constraints, hence cannot be selected by the minimization of any linear function defined on this polytope. Nevertheless, we shall show they do exist and are selected by the minimization of a specific strictly convex function introduced below.
} 
Instead, stochasticity restores uniqueness without the need for a genericity assumption in our model - and indeed in the more general setting studied by Galichon and Salanié [15].

Second, an affirmative explicit solution to the Choo-Siow Inverse Problem makes the Choo-Siow model useful in econometric analysis. The matrix $\Pi$ is exogenous and unobserved in data, but can be point-estimated from an observed marriage distribution. An economic or social shock will affect the systematic utilities that agents of various types incur by marrying agents of various others, and will therefore alter the value of $\Pi$. This effect can be approximated to form an updated matrix of aggregated systematic parameters $\Pi^{\prime}$. Existence and uniqueness guarantee that there will be exactly one marriage distribution that results from the shock, making the model predictive. In the same vein, demographers are often interested in predicting how marriage distributions will change due to changing demographics, i.e. changes in the population vector $\nu$. Our closed form solution makes it possible to compute the sign and in some cases the magnitude of such changes explicitly.

Finally, if the model can be shown to admit a unique distribution, the estimated parameters $\pi_{i j}$ are an alternative characterization of the observed marriage distribution. The recharacterization is useful because the parameters of the Choo-Siow model have a behavioural interpretation, and are not merely observed data.

2.3. Summary of progress and related literature. The related local uniqueness question was resolved by Choo and Siow in [7]. However the issue of global uniqueness was left open, and posed as an open problem in a subsequent working paper by Siow [23. We resolve this question positively by introducing a variational principle and a change of variables which allows us to exploit convexity. The question of existence of $\left(\mu_{i j}\right)$ for all $\Pi=\left(e^{\pi_{i j}}\right)$ was addressed in a working paper of Choo, Seitz and Siow [6] by appealing to the Tarski fixed point theorem; see also the related results of Fox [12] and Dagsvik [8]. However the proofs there are long and involved, whereas the variational proof in the present paper is simple and direct and follows from continuity and compactness by way of an elementary estimate. Moreover, it leads to an explicit representation of the solution. This allows us to rigorously confirm various desirable and intuitive features of Choo-Siow matching, whose presence or absence might in principle be used as a test to refute the validity of various alternative matching models. Among other results, we show for example that an increase in the number of men of a given type increases the equilibrium transfer paid by such men to their spouses, while also increasing the percentage of such men who choose to remain single. See Theorem 2 below for related statements and more surprising conclusions. Independently of us, Galichon and Salanié [14] [15] developed a variational approach which extends the Choo-Siow model by allowing for randomness of much more general form. From theoretical considerations, they derive a closed-form expression for a strictly concave social welfare function governing competitive equilibria in their models, and identities relating different sets of endogenous variables under consideration. This gives an alternate approach to existence and uniqueness of equilibria in our setting as a special case. Apart from its convex analytic nature, their approach is quite distinct from ours, and leads to very different insights and interpretations. 


\section{Precise Statement of Results}

In the preceding remarks, the Choo-Siow inverse problem was phrased in terms of finding existence and uniqueness of equilibrium $\mu$ given exogenous data $\Pi$ and $\nu$. As the name suggests, it is also useful to think of this problem as one of inverting a function. From this point of view, even though $\Pi$ is exogenous, we may prefer instead to consider $\Pi$ as the image of a marriage distribution under a transformation that we seek to invert.

Remark 1 (Incomplete participation). From the market equilibrium point of view, the fact that the left hand-side of (9) becomes infinite when $\mu_{i 0}$ or $\mu_{0 j}$ is equal to zero is unproblematic. It means that for no finite value of the exogenous $\Pi$ is sufficient to induce all the representatives of some type to marry. However from the inverse problem point of view, it is necessary to stipulate that $\mu_{i 0}$ and $\mu_{0 j}$ be strictly positive.

3.1. Preliminaries. Let us begin with a reformulation of the Choo-Siow inverse problem; Siow attributes this reformulation to Angelo Melino. Let $\beta_{i}:=\mu_{i 0}$ and $\beta_{I+j}:=\mu_{0 j}$ denote the number of unmarried men and women of types $i=1, \ldots, I$ and $j=1, \ldots, J$ respectively. Since the gains matrix (9) can be used to express each component $\mu_{i j}=\beta_{i} \beta_{I+j} \Pi_{i j}$ of the marital distribution in terms of these new variables, the population constraints (10) - (11) can be reduced to a system

$$
\begin{aligned}
\beta_{i}^{2}+\sum_{j=1}^{J} \beta_{i} \beta_{I+j} \Pi_{i j}-\nu_{i}=0, & 1 \leq i \leq I, \\
\beta_{I+j}^{2}+\sum_{i=1}^{I} \beta_{i} \beta_{I+j} \Pi_{i j}-\nu_{I+j}=0, & 1 \leq j \leq J .
\end{aligned}
$$

of $(I+J)$ quadratic polynomials in the $(I+J)$ variables $\left\{\beta_{k}\right\}_{k=1}$ counting the number of umarried men and women of each type.

A solution to this system of equations is a vector of amplitudes $\beta$ that has $(I+J)$ components. Abstractly, its components might be real, complex, or both. The Choo-Siow Inverse Problem is equivalent to showing that the polynomial system (13) has a unique solution with real positive amplitudes for all gains matrices $\Pi$ and population vectors $\nu=[m \mid f]$ with positive components. The full marital distribution satisfying (9)-(12) is then recovered by choosing $\mu_{i j}=\beta_{i} \beta_{I+j} \Pi_{i j}$. Our proof is variational. We construct a functional $E(\beta)$ with the property that $\beta$ is a critical point of $E$ - meaning a point where $E$ has zero derivative - if and only if $\beta$ satisfies equation (13). We then show that $E$ has exactly one critical point in the positive orthant $\left(\mathbf{R}_{+}\right)^{I+J}$, and give a formula for this critical point using the Legendre transform of a related function.

The main result of this paper is the following theorem, which solves the Choo-Siow Inverse problem.

Theorem 1 (Existence, uniqueness, and explicit representation of a real positive solution). If all the entries of $\Pi=\left(\Pi_{i j}\right)$ are non-negative, and those of $\nu=[m \mid f]$ are strictly positive, then precisely one solution $\beta$ of (13) lies in the positive orthant of $\mathbf{R}^{I+J}$. Indeed, 
the solution $b:=\left(\log \beta_{1}, \ldots, \log \beta_{I+J}\right)$ satisfies $b=(D H)^{-1}(\nu)=D H^{*}(\nu)$ where $H(b)$ and $H^{*}(\nu)$ are smooth strictly convex dual functions on $\mathbf{R}^{I+J}$ defined by

$$
H(b):=\frac{1}{2} \sum_{k=1}^{I+J} e^{2 b_{k}}+\sum_{i=1}^{I} \sum_{j=1}^{J} \Pi_{i j} e^{b_{i}+b_{I+j}}
$$

and

$$
H^{*}(\nu):=\sup _{b \in \mathbf{R}^{I+J}}\langle\nu, b\rangle-H(b) .
$$

Here $\langle\cdot, \cdot\rangle$ denotes the inner product on $\mathbf{R}^{I+J}$.

Remark 2 (Unpopulated types). In case $m_{i}=0$ or $f_{j}=0$, we simply reformulate the problem in fewer than $I+J$ variables, corresponding only to the populated types. This reformulation shows the conclusions of Theorem 1 extend also to population vectors $\nu=$ $[m \mid f]$ whose entries are merely non-negative, instead of strictly positive.

Since each matrix $\left(\mu_{i j}\right)$ with non-negative entries solving (9)-(12) corresponds to a solution $\beta$ of (13) having positive amplitudes $\beta_{i}=\sqrt{\mu_{i 0}}$ and $\beta_{I+j}=\sqrt{\mu_{0 j}}$, this theorem gives the sought characterization of $\left(\mu_{i j}\right)$ by $\Pi$. Moreover, this characterization facilitates computing variations in the marital arrangements in response to changes in the data $(\Pi, \nu)$ :

Theorem 2 (Comparative statics). Let the unique solution to the Choo-Siow inverse problem with exogenous data $\Pi$ and $\nu$ be given by $\beta(\Pi, \nu)$. Then (a) the percentage change of singles $\beta_{k}^{2}$ with respect to the population parameter $\nu_{\ell}$ turns out to define a symmetric and positive definite matrix

$$
r_{k \ell}:=\frac{1}{\beta_{k}^{2}} \frac{\partial \beta_{k}^{2}}{\partial \nu_{\ell}}
$$

here $k, \ell \in\{1, \ldots, I+J\}$. This symmetric positive-definiteness implies, among other things, the expected monotonicity $r_{k k}>0$, the unexpected symmetry $r_{k \ell}=r_{\ell k}$, and more subtle constraints relating these percentage rates of change and the corresponding substitution effects such as $\left|r_{k \ell}\right|<\sqrt{r_{k k} r_{\ell \ell}}$.

(b) Additionally we can account for the sign, and in some cases bound the magnitude, of each entry of the matrix $R=\left(r_{k \ell}\right)$. To avoid trivialities, assume no column or row of $\Pi$ vanishes, so no observable type of individual is compelled to remain single. Then,

$$
r_{k \ell}<0
$$

if $k \in\{1 \ldots I\}$ and $\ell \in\{I+1 \ldots I+J\}$ (or vice versa). Second, if $k, \ell \in\{1 \ldots I\}$, then

$$
\frac{1}{2}\left(\beta_{k}^{2}+\nu_{k}\right) r_{k \ell} n>\delta_{k \ell}:= \begin{cases}0 & \text { if } k \neq \ell \\ 1 & \text { otherwise. }\end{cases}
$$

Similarly, (18) also holds if both $k, \ell \in\{I+1 \ldots I+J\}$.

These qualitative comparative statics have a simple interpretation. Increased supply of any type $k$ of man coaxes more women into marriage (17) and decreases the number of men who wish to marry. The last statement of the theorem says that this decrease is not 
merely due to the fact that there are more men. Rather, men of type $\ell \neq k$ who would have chosen marriage under the old regime choose to be single after the shock (18). The following corollary explains how the additional women are coaxed into marriage: it shows increased competition among men leads to larger equilibrium transfers to their female spouses, as is further explained in subsection $\$ 7$. The corollary asserts not only monotonicity of utility transferred by men of type $i$, but also of the percentage who choose to remain single, as a function of their abundance in the population.

Corollary 3 (Utility transferred and non-participant fraction increase with abundance). For all $i \leq I, j \leq J$, and $k \leq I+J$, with the hypotheses and notation of Theorem 2 ,

$$
\begin{aligned}
\frac{\partial}{\partial \nu_{i}}\left(\eta_{i j}^{f}-\eta_{i j}^{m}\right) & >0 \\
\text { and } \frac{\partial}{\partial \nu_{k}}\left(\frac{\beta_{k}^{2}}{\nu_{k}}\right) & >0 .
\end{aligned}
$$

\section{Derived Statics AND FURTher COnJeCtures}

It is possible to express many quantities of interest in terms of the symmetric positivedefinite matrix $R=\left(r_{i j}\right)$ from Theorem 2, whose entries encode the relative change in the number of type $i$ individuals who choose not to marry in response to a fluctuation in the total number $\nu_{j}$ of type $j$ individuals in the population. For example, in the Choo-Siow model, the number of marriages $\mu_{i j}$ of type $i$ men to type $j$ women is given by the geometric mean (9) of the number of singles of the two given types times the corresponding entry in the gains matrix: $\mu_{i j}=\Pi_{i j}\left(\mu_{i 0} \mu_{0 j}\right)^{1 / 2}=\Pi_{i j} \beta_{i} \beta_{j}$. Since $\Pi_{i j}$ is exogenous, we immediately obtain a formula

$$
\frac{\partial \log \mu_{i j}}{\partial \nu_{k}}=\frac{1}{2}\left(r_{i k}+r_{k, I+j}\right)
$$

showing the relative change in the number of type $(i, j)$ marriages caused by fluctuations in the total population of type $k$ individuals is just the average of the relative changes $r_{i k}:=2 \partial\left(\log \beta_{i}\right) / \partial \nu_{k}$ and $r_{I+j, k}$ in the numbers of unmarrieds of the corresponding types $i$ and $j$.

We may also consider fluctuations in the number of singles of type $k$ in response to changes in the exogenous gains parameters $\Pi_{i j}$ when the population $\nu$ of each type of man and woman is held fixed. In section 7.2, the implicit function theorem is used to derive

$$
\frac{\partial \beta_{k}}{\partial \Pi_{i j}}=-\beta_{i} \beta_{I+j}\left(\frac{\partial \beta_{k}}{\partial \nu_{i}}+\frac{\partial \beta_{k}}{\partial \nu_{I+j}}\right)
$$

for all $i \in\{1, \ldots, I\}, j \in\{1, \ldots, J\}$, and $k \in\{1, \ldots, I+J\}$, or equivalently

$$
\frac{\partial \log \beta_{k}}{\partial \prod_{i j}}=-\frac{\mu_{i j}}{2 \prod_{i j}}\left(r_{k i}+r_{k, I+j}\right)
$$

The equation (22) has an intuitive interpretation. An increase in the total systematic gains to an $(i, j)$ marriage (produced, for example, by an isolated increase in the value of type $j$ marriages to type $i$ men, or an isolated decrease in the value of remaining single) has 
the same effect as decreasing the supply of the men or women of the respective types by a proportionate amount, weighted by the geometric mean of the unmarried men and women of type $i$ and $j$.

Now Theorem 2 shows the the summands above to have opposite signs, so the sign of their sum $r_{k i}+r_{k, I+j}$ will fluctuate according to market conditions. If $k=i$ or $k=I+j$ however, it is natural to conjecture that this sum is positive, in which case (21) shows the the number of type $(i, j)$ marriages $\mu_{i j}$ would be an increasing function of the population size of type $i$ (and independently, type $j$ ) individuals. Similarly (23) then asserts the number of type $i$ and $j$ singles to be a decreasing function of the total gains $\Pi_{i j}$ for type $(i, j)$ marriages. This conjecture amounts to requiring each positive diagonal entry $r_{k k}$ in the matrix $R$ to dominate each negative entry $-r_{k \ell}$ in its row or column - a plausible strengthening (whose proof, alas, eludes us) of the claim $r_{k \ell}^{2} \leq r_{k k} r_{\ell \ell}$ established in Theorem 2(a).

4.1. Summary of comparative statics. Before turning to the proof of the theorems listed above, let us conclude by recapping our comparative statics.

- Increasing the men of a given type increases the number of singles of all male types, and decreases the number of singles of all female types.

- Increasing the number of men of a given type increases the transfer they must pay to any woman they marry (19).

- The percentage rate of change of unmarried men of type $i$ due to increases in women of type $j$ is equal to the percentage rate of change of unmarried women of type $j$ due to increases in men of type $i$.

- The marital participation rate $\sum_{j=1}^{J} \frac{\mu_{i j}}{m_{i}}$ decreases with an increase in own type $m_{i}$.

\section{A New Variational Principle (Proof of Theorem 1)}

5.1. Variational method: existence of a solution. Consider the function $E: \mathbf{R}^{I+J} \rightarrow$ $\mathbf{R} \cup\{+\infty\}$, defined as follows:

$$
E(\beta):=\frac{1}{2} \sum_{k=1}^{I+J} \beta_{k}^{2}+\sum_{i=1}^{I} \sum_{j=1}^{J} \Pi_{i j} \beta_{i} \beta_{I+j}-\sum_{k=1}^{I} \nu_{k} \log \left|\beta_{k}\right| .
$$

It diverges to $+\infty$ on the coordinate hyperplanes where the $\beta_{k}$ vanish, but elsewhere is smooth.

We differentiate and observe that $\beta$ is a critical point of $E$ if and only if (13) holds. Notice strict positivity of the components of $\nu=[m \mid f]$ implies the corresponding component of a solution $\beta$ to (13) is non-vanishing, hence no solutions occur on the coordinate hyperplanes which separate the different orthants. In words, the critical points of $E$ are precisely those that satisfy the system of equations we wish to show has a unique real positive root. It therefore suffices to show that $E(\beta)$ has a unique real positive critical point; for then (13) admits exactly one real positive solution. Let us show at least one such solution exists, by showing $E(\beta)$ has at least one critical point: namely, its minimum in the positive orthant. 
Claim 4 (Existence of a minimum). If all the entries of $\Pi=\left(\Pi_{i j}\right)$ are non-negative, and those of $\nu=[m \mid f]$ are strictly positive, the function $E(\beta)$ on the positive orthant defined by (24) attains its minimum value.

Proof. Since $E(\beta)$ is continuous, the claim will be established if we show the sublevel set $B_{\lambda}:=\left\{\beta \in\left(\mathbf{R}_{+}\right)^{I+J} \mid E(\beta) \leq \lambda\right\}$ is compact for each $\lambda \in \mathbf{R}$. Non-negativity of $\Pi_{i j}$ combines with positivity of $\nu_{k}, \beta_{k}$, and the inequality $\log \beta_{k} \leq \beta_{k}-1$ to yield

$$
\begin{aligned}
E(\beta) & \geq \sum_{k=1}^{I+J} \frac{1}{2} \beta_{k}^{2}-\nu_{k}\left(\beta_{k}-1\right) \\
& =\frac{1}{2} \sum_{k=1}^{I+J}\left(\beta_{k}-\nu_{k}\right)^{2}-\left(\nu_{k}-1\right)^{2}+1 .
\end{aligned}
$$

It follows that $B_{\lambda}$ is bounded away from infinity. Since $E(\beta)$ diverges to $+\infty$ on the coordinate hyperplanes, it follows that $B_{\lambda}$ is also bounded away from the coordinate hyperplanes - hence compactly contained in the positive orthant.

5.2. Uniqueness, convexity, and Legendre transforms. With this critical point characterization of the solution in mind, let us observe for $\beta \in \mathbf{R}^{I+J}$ in the positive orthant, defining $b_{k}:=\log \beta_{k}$ implies $E(\beta)=H(b)-\langle\nu, b\rangle$, where $H(b)$ is defined in (14). Since the change of variables $\beta_{k} \in \mathbf{R}_{+} \longmapsto b_{k}=\log \beta_{k} \in \mathbf{R}$ is a diffeomorphism, it follows that critical points of $H(b)-\langle\nu, b\rangle$ in the whole space $\mathbf{R}^{I+J}$ are in one-to-one correspondence with critical points of $E(\beta)$ in the positive orthant.

On the other hand, $H(b)$ is manifestly convex, being a non-negative sum of convex exponential functions of the real variables $b_{k}$; in fact $\Pi_{i j} \geq 0$ shows the Hessian $D^{2} H(b)$ dominates what it would be in case $\Pi=0$, namely the diagonal matrix with positive entries $\operatorname{diag}\left[2 e^{2 b_{1}}, \ldots, 2 e^{2 b_{I+J}}\right]$ along its diagonal. Thus $H(b)$ is strictly convex throughout $\mathbf{R}^{I+J}$, and $E(\beta)=H(b)-\langle\nu, b\rangle$ can admit only one critical point $\beta$ in the positive orthant - the minimizer whose existence we have already shown. The solution $\beta$ to (13) which we seek therefore coincides with the unique point at which the maximum is attained.

This last fact means that $b$ maximizes the right-hand side of the following equation:

$$
\begin{aligned}
H^{*}(\nu) & :=\sup _{b \in \mathbf{R}^{I+J}}\langle\nu, b\rangle-H(b) \\
& =\sup _{\beta \in\left(\mathbf{R}_{+}\right)^{I+J}}-E(\beta) .
\end{aligned}
$$

The function $H^{*}$ defined pointwise by the above equation is the Legendre transform or convex dual function of $H$; see Appendix $\mathrm{B}$ for details. It follows that the solution $b$ satisfies $\nu=D H(b)$. Thus $b=D H^{*}(\nu)$ by the duality of $H$ and $H^{*}$. This provides an explicit formula for $b$ in terms of the derivative of $H^{*}$. 


\section{Comparative Statics (Proof of Theorem 2)}

6.1. Positive definiteness (a). Our representation of the solution in terms of the Legendre transform of the convex function $H$ can be used to obtain information about the derivatives of the solutions with respect to the population parameters $\nu$.

Suppose we wish to know how the number of marriages $\mu_{i j}=\Pi_{i j} \beta_{i} \beta_{I+j}$ of each type $(i, j)$ varies in response to slight changes in the population vector $\nu$, assuming the gains matrix $\Pi$ remains fixed. This is easily computed from the percentage rate of change $r_{k \ell}$ in the number $\beta_{k}^{2}$ of unmarrieds of each type, which is given in terms of the Hessian of either (14) or (15) by

$$
r_{k \ell}:=\frac{1}{\beta_{k}^{2}} \frac{\partial \beta_{k}^{2}}{\partial \nu_{\ell}}=2 D_{k \ell}^{2} H^{*}(\nu)=2\left(\left.D^{2} H\right|_{\left(\log \beta_{1}, \ldots, \log \beta_{I+J}\right)} ^{-1}\right)_{k \ell}, \quad 1 \leq k, \ell \leq I+J .
$$

To see that these equalities hold, observe that the solution $\beta$ is the point where the maximum (15) is attained. The Legendre transform $H^{*}(\nu)$ of $H$ defined by this maximum is manifestly convex, and its smoothness is well-known to follow from the positive-definiteness of $D^{2} H(b)>0$ as in Lemma 9. Moreover $b=D H^{*}(D H(b))$, whence the maximum (15) is attained at $b=D H^{*}(\nu)$ and $D^{2} H(b)^{-1}=D^{2} H^{*}(D H(b))=D^{2} H^{*}(\nu)>0$. This positive definiteness implies the first half of the Theorem 2 .

6.2. Qualitative characterization of comparative statics (b). To complete our qualitative description of the substitution effects in this section, we apply the following theorem from functional analysis to matrices $T: \mathbf{R}^{n} \longrightarrow \mathbf{R}^{n}$. We define the operator norm of such a matrix by $\|T\|_{o p}:=\max _{0 \neq v \in \mathbf{R}^{n}}|T(v)| /|v|$, where $|v|=\langle v, v\rangle^{1 / 2}$ denotes the Euclidean norm.

Theorem 5 (Neumann series for the resolvent of a linear contraction). If $\|T\|_{o p}<1$ for $T: \mathbf{R}^{n} \longrightarrow \mathbf{R}^{n}$, the operator $(1-T)^{-1}$ exists and is equal to $\sum_{k=0}^{\infty} T^{k}$.

Next, we consider the matrix $\left.D^{2} H(b)\right|_{\left(\log \beta_{1}, \ldots, \log \beta_{I+J}\right)}$, and derive properties of its inverse, whose entries give the various values of $r_{k \ell} / 2$. Differentiating the known function $H(b)$ twice yields a positive-definite $(I+J) \times(I+J)$ matrix which can be factored into the form

$$
2 R^{-1}=\left.D^{2} H\right|_{b=\left(\log \beta_{1}, \ldots, \log \beta_{I+J}\right)}=\Delta\left(\begin{array}{cc}
\Delta_{I} & \Pi \\
\Pi^{T} & \Delta_{J}
\end{array}\right) \Delta
$$

where $\Delta=\operatorname{diag}\left[e^{b_{1}}, \ldots, e^{b_{I+J}}\right]=\operatorname{diag}[\beta]$, while $\Delta_{I}$ and $\Delta_{J}$ are $I \times I$ and $J \times J$ diagonal submatrices whose diagonal entries are all larger than two:

$$
\begin{aligned}
& \left(\Delta_{I}\right)_{i i}=2+\frac{1}{\beta_{i}^{2}} \sum_{j=1}^{J} \Pi_{i j} \beta_{i} \beta_{I+j}=1+\frac{\nu_{i}}{\beta_{i}^{2}}, \\
& \left(\Delta_{J}\right)_{j j}=2+\frac{1}{\beta_{I+j}^{2}} \sum_{i=1}^{I} \Pi_{i j} \beta_{i} \beta_{I+j}=1+\frac{\nu_{I+j}}{\beta_{I+j}^{2}} .
\end{aligned}
$$

Here we have used the fact that the values $\beta$ are critical points and therefore satisfy the first order conditions (13) to simplify these diagonal terms. 
There are determinant and inverse formulae for block matrices which assert [17] that

$$
\operatorname{det}\left(\begin{array}{cc}
\Delta_{I} & \Pi \\
\Pi^{T} & \Delta_{J}
\end{array}\right)=\operatorname{det}\left(\Delta_{I}\right) \operatorname{det}\left(\Delta_{J}\right) \operatorname{det}\left(1-\Delta_{I}{ }^{-1} \Pi \Delta_{J}{ }^{-1} \Pi^{T}\right)
$$

and

$$
\left(\begin{array}{cc}
\Delta_{I} & \Pi \\
\Pi^{T} & \Delta_{J}
\end{array}\right)^{-1}=\left(\begin{array}{cc}
\left(\Delta_{I}-\Pi \Delta_{J}^{-1} \Pi^{T}\right)^{-1} & -\left(\Delta_{I}-\Pi \Delta_{J}^{-1} \Pi^{T}\right)^{-1} \Pi \Delta_{J}{ }^{-1} \\
-\Pi^{T} \Delta_{I}{ }^{-1}\left(\Delta_{J}-\Pi^{T} \Delta_{I}^{-1} \Pi\right)^{-1} & \left(\Delta_{J}-\Pi^{T} \Delta_{I}^{-1} \Pi\right)^{-1}
\end{array}\right) .
$$

The determinant (30) is positive by (29) and Theorem 1. We will now show that the eigenvalues of the matrix $A(s)=\Delta_{I}{ }^{-1} s \Pi \Delta_{J}{ }^{-1} s \Pi^{T}$, appearing in (30)-(31) are bounded above by 1 and below by -1 for all values of $s \in[0,1]$. This will have implications respecting the signs of the entries of (31), whose $(k, \ell)^{t h}$ entry is in fact equal to $\beta_{k} \beta_{\ell} r_{k \ell} / 2$ hence shares the sign of the change (17)-(18) which we desire to estimate. Namely, it will allow us to apply Theorem 5 to block entries such as $\left(\Delta_{I}-\Pi \Delta_{J}{ }^{-1} \Pi^{T}\right)^{-1}=(1-A(1))^{-1} \Delta_{I}{ }^{-1}$ in (31).

Let $\lambda^{\max }(s)$ be the largest eigenvalue of $A(s)$. Then, the smallest eigenvalue of $(1-A(s))$ is equal to $\left(1-\lambda^{\max }(s)\right)$. We proceed by continuously deforming from $s=0$ to $s=1$ : The eigenvalues of $(1-A(0))$ are equal to 1 , as $A(0)$ is in fact equal to the zero matrix. Since $\operatorname{det}(1-A(s))>0$ for all $s \in[0,1]$, continuity of $\lambda^{\max }(s)$ and the intermediate value theorem imply that $1-\lambda^{\max }(s)>0$ for all $s$, so that $\lambda^{\max }(1)<1$. Since no row of $\Pi$ vanishes, $A(s)$ has positive entries whenever $s>0$. The Perron-Frobenius theorem therefore implies that any negative eigenvalue $\lambda$ of $A(1)$ is bounded by $|\lambda|<\lambda^{\max }(1)$.

Since $A$ has positive entries and $\|A\|_{o p}<1$, Theorem 5 indicates that the entries of $(1-A)^{-1}$ are all positive - exceeding one on the diagonal. But $\beta_{k} \beta_{\ell} r_{k \ell} / 2$ coincides with the $(k, \ell)^{t h}$ entry of $(1-A)^{-1} \operatorname{diag}\left[\beta_{1}^{2} /\left(\beta_{1}^{2}+\nu_{1}\right), \ldots, \beta_{I}^{2} /\left(\beta_{I}^{2}+\nu_{I}\right)\right]$, giving the desired inequalities (18) whenever $k, \ell \in\{1, \ldots, I\}$. The signs of the remaining derivatives (17)(18) may be verified by applying the same technique to the three other submatrices present in (31), thus completing the proof of Theorem 2(a)-(b).

\section{Transfer utilities (Proof of Corollary 3 and subsequent remarks)}

7.1. Varying the population vectors $\nu$. Given a specification of $\Pi$ and $\nu=\left[m_{i} \mid f_{j}\right]$, the Choo-Siow model predicts a unique vector $\beta=\left[\mu_{i 0} \mid \mu_{0 j}\right]$ of unmarrieds. Given a fixed $\Pi$ and a fixed $\beta$, the full marriage distribution can then be uniquely recovered. It is therefore possible to view $\mu$ as a single valued (smooth) function of $\Pi$ and $\nu$. By Theorem 2 , the signs of $r_{k \ell}$ are independent of $\Pi$ and $\nu$ and depend only on whether $k \in\{1, \ldots, I\}$, or $k \in\{I+1, \ldots, I+J\}$, and likewise for $\ell$. It is perhaps useful to visualize these comparative statics as the entries of the matrix $D \beta$ with $D_{\ell} \beta_{k}:=\frac{\partial \beta_{k}}{\partial \nu_{\ell}}$. Then, $D \beta$ is a block matrix that is positive in its upper-left and lower-right blocks, and negative in its upper-right and lower-left blocks. Schematically, (17)-(18) yield

$$
D \beta=\left(\begin{array}{c}
+- \\
-+
\end{array}\right) .
$$


Reverting back to the Choo-Siow notation for unmarrieds and population vectors, we have for all $k$ and $\ell$ :

$$
\frac{\partial \mu_{k 0}}{\partial m_{\ell}}>0, \quad \frac{\partial \mu_{k 0}}{\partial f_{\ell}}<0, \quad \frac{\partial \mu_{0 k}}{\partial f_{\ell}}>0, \quad \frac{\partial \mu_{0 k}}{\partial m_{\ell}}<0 .
$$

These basic comparative statics yield qualitative information about other more complex quantities of interest. As indicated following equation (7), the quantity $\eta_{i j}^{m}+\eta_{i j}^{f}-\eta_{i 0}^{m}-\eta_{0 j}^{f}$ is exogenous, whereas the first two individual summands are separately endogenous and determined within the model. In the original formulation of this model, present in [7, our endogenous payoff $\eta_{i j}^{m}=\tilde{\eta}_{i j}^{m}-\tau_{i j}$ is separated into a systematic return $\tilde{\eta}_{i j}^{m}$ presumed to be exogenous, and a utility transfer $\tau_{i j}$ from husband to wife, which is endogenous and set in equilibrium. Similarly, $\eta_{i j}^{f}=\tilde{\eta}_{i j}^{f}+\tau_{i j}$.

In equilibrium (66), both of the following equations hold:

$$
\begin{aligned}
& \log \left(\mu_{i j}\right)-\log \left(\mu_{0 j}\right)=\eta_{i j}^{f}-\eta_{0 j}^{f}=\tilde{\eta}_{i j}^{f}+\tau_{i j}-\tilde{\eta}_{0 j}^{f}, \\
& \log \left(\mu_{i j}\right)-\log \left(\mu_{i 0}\right)=\eta_{i j}^{m}-\eta_{i 0}^{m}=\tilde{\eta}_{i j}^{m}-\tau_{i j}-\tilde{\eta}_{i 0}^{m} ;
\end{aligned}
$$

there is no utility transferred by remaining single. Subtracting one from the other, we see that:

$$
\log \left(\frac{\mu_{i 0}}{\mu_{0 j}}\right)=2 \tau_{i j}+c_{i j}
$$

where $c_{i j}=\left(\tilde{\eta}_{i j}^{f}-\tilde{\eta}_{0 j}^{f}-\tilde{\eta}_{i j}^{m}+\tilde{\eta}_{i 0}^{m}\right)$ is exogenous.

We denote the differentiation operator $\frac{\partial}{\partial \nu_{k}} f$ by $\dot{f}$ (suppressing the dependence on $k$ ). Differentiating $c_{i j}=\left(\eta_{i j}^{f}-2 \tau_{i j}-\tilde{\eta}_{0 j}^{f}-\eta_{i j}^{m}+\tilde{\eta}_{i 0}^{m}\right)$ and (36) yields:

$$
\frac{\partial}{\partial \nu_{k}}\left(\eta_{i j}^{f}-\eta_{i j}^{m}\right)=2 \dot{\tau_{i j}}=\frac{\dot{\mu_{i 0}}}{\mu_{i 0}}-\frac{\dot{\mu_{0 j}}}{\mu_{0 j}}
$$

The inequalities (33) now determine the sign of $\tau_{i j}$, which depends on the differentiation variable $\nu_{k}$. Since $\dot{\mu_{i 0}}$ and $\mu_{0 j}$ have opposite signs, according to Theorem 2, we find

$$
\frac{\partial \tau_{i j}}{\partial m_{i}}>0
$$

which means the transfer of type $i$ men to each type of spouse must increase in response to an isolated increase in the population of men of type $i$. This is expected because an increase in the number of type $i$ men introduces additional competition for each type of women, due to the smearing present in the model. To decrease the number of type $i$ men demanding marriage to a particular type of woman to a level that permits one-to-one matching requires an increase in the transfer to crowd out some men.

While in principle the men might re-distribute so that the proportion of married men remains the same, our next computation shows this is not the case. We consider the marital participation rate of type $k$ individuals, or rather the non-participation rate $s_{k}(\nu):=\beta_{k}^{2} / \nu_{k}$, 
defined as the proportion of individuals who choose not to marry. Differentiation yields

$$
\begin{aligned}
\frac{\partial s_{k}}{\partial \nu_{k}} & =\frac{\beta_{k}^{2}}{\nu_{k}^{2}}\left(\nu_{k} r_{k k}-1\right) \\
& >\frac{\beta_{k}^{2}}{\nu_{k}^{2}}\left(\frac{\nu_{k}-\beta_{k}^{2}}{\nu_{k}+\beta_{k}^{2}}\right),
\end{aligned}
$$

according to (18). But this is manifestly positive since the number $\beta_{k}^{2}$ of singles of type $k$ cannot exceed the total number $\nu_{k}$ of type $k$ individuals. This means, for example, that an increase in the total population of type $k$ men increases the percentage of type $k$ men who choose to remain unmarried, given a fixed population of women and men of other types (and assuming, as always, that the exogenous gains matrix $\Pi$ remains fixed). It concludes the proof of Corollary 3 .

7.2. Varying the gains data $\Pi$ (Proof of (22)-(23)). The population vector $\nu$ is one variable of interest. However the function $\beta$ also depends on the gains parameters $\Pi_{i j}$. The complete derivative $D_{(\nu, \Pi)} \beta=\left[D_{\nu} \beta \mid D_{\Pi} \beta\right]$ is an $(I+J) \times(I J+I+J)$ matrix. As such there are linear dependencies among its rows and columns. Since the matrix $D_{\nu} \beta$ is invertible, its columns are linearly independent and form a basis of the column space. Hence, the remaining columns of the complete derivative can be expressed using linear combinations of them. The implicit function theorem applied to this problem turns out to yield the simple linear relationship (22):

$$
\frac{\partial \beta_{k}}{\partial \Pi_{i j}}=-\beta_{i} \beta_{I+j}\left(\frac{\partial \beta_{k}}{\partial \nu_{i}}+\frac{\partial \beta_{k}}{\partial \nu_{I+j}}\right)
$$

for all $i \in\{1, \ldots, I\}, j \in\{1, \ldots, J\}$, and $k \in\{1, \ldots, I+J\}$.

Equilibrium (13) coincides with vanishing of the function $F(\beta, \nu, \Pi): \mathbf{R}^{(I+J)+(I+J)+(I J)} \rightarrow$ $\mathbf{R}^{I+J}$ defined by

$$
\begin{array}{cc}
F_{i}(\nu, \Pi)=\beta_{i}^{2}+\sum_{j=1}^{J} \beta_{i} \beta_{I+j} \Pi_{i j}-\nu_{i}, & 1 \leq i \leq I \\
F_{j}(\nu, \Pi)=\beta_{I+j}^{2}+\sum_{i=1}^{I} \beta_{i} \beta_{I+j} \Pi_{i j}-\nu_{I+j}, & 1 \leq j \leq J .
\end{array}
$$

The implicit function theorem stipulates that if the derivative $\left.D_{\beta} F\right|_{\beta_{0}, \nu_{0}, \Pi_{0}}$ is invertible, there is a small neighbourhood around $\left(\beta_{0}, \nu_{0}, \Pi_{0}\right)$ inside which for each $(\nu, \Pi)$ there is a unique $\beta$ satisfying equation (13), and further that $\beta$ depends smoothly on $(\nu, \Pi)$. The implicit function theorem also provides a formula for the derivative of the implicit function $\beta(\nu, \Pi)$. It is obtained by applying the chain-rule to $F(\beta(\nu, \Pi), \nu, \Pi)$ :

$$
\left[D_{\nu} \beta \mid D_{\Pi} \beta\right]_{\nu_{0}, \Pi_{0}}=-\left[D_{\beta} F\right]^{-1}\left[D_{\nu} F \mid D_{\Pi} F\right]_{\beta_{0}, \nu_{0}, \Pi_{0}} .
$$

Since $\frac{\partial F_{k}}{\partial \nu_{\ell}}=-\delta_{k \ell}$, and $\frac{\partial F_{\ell}}{\partial \Pi_{i j}}=\beta_{i} \beta_{I+j}\left(\delta_{i \ell}+\delta_{I+j, \ell}\right)$, the first part of the preceding formula yields $\left[D_{\beta} F\right]^{-1}=D_{\nu} \beta$, and the second part then implies (38). Theorem 2 shows $D_{\nu} \beta$ is 
invertible, so the hypotheses of the implicit function theorem are globally satisfied and our calculations are valid. This concludes the proof of (22)-(23).

\section{Appendix A. Derivation of the preference probabilities}

The random variable present in the definition of male and female utility is the Gumbel extreme value distribution, introduced to the economics literature by McFadden [19]:

Definition 6 (Gumbel distribution). A random variable $\epsilon$ is Gumbel if it has cumulative distribution function $F(\epsilon)=\exp (-\exp (-\epsilon))$.

Here $\operatorname{Pr}(\epsilon<x)=F(x)$ gives the probability that the realization of this random variable takes a value less $x \in \mathbf{R}$. The corresponding density function is $F^{\prime}(x)=f(x)=\exp (-(x+$ $\exp (-x))$. The mean of $\epsilon$ is the Euler-Mascheroni constant, which is approximately equal to $\gamma=0.57 \ldots$ Its variance is equal to $\frac{\pi^{2}}{6}$.

We now use this distribution to derive the discrete probability distribution (2).

Lemma 7. Suppose $\sigma>0$ and $\eta_{i j} \in \mathbf{R}$ are constants, while for each choice of $j=$ $0, \ldots, J$, the $\epsilon_{i j g}$ are independent identically distributed random variables with the Gumbel distribution. Then

$$
\operatorname{Pr}\left(\eta_{i j}+\epsilon_{i j g}=\max _{0 \leq k \leq J} \eta_{i k}+\epsilon_{i k g}\right)=\frac{\exp \left(\frac{\eta_{i j}}{\sigma}\right)}{\sum_{k=0}^{J} \exp \left(\frac{\eta_{i k}}{\sigma}\right)} .
$$

Proof. It costs no generality to assume $\sigma=1$. Then

$$
P:=\operatorname{Pr}\left(\eta_{i j}+\epsilon_{i j g} \geq \eta_{i k}+\epsilon_{i k g} \forall k\right)=\int_{-\infty}^{\infty} d \epsilon F^{\prime}(\epsilon) \Pi_{k \neq j} F\left(\eta_{i j}+\epsilon-\eta_{i k}\right) .
$$

This formula follows from Bayes' rule for conditional probability, and independence of the various random variables involved. Substituting in the explicit formula for the Gumbel distribution from Definition 6 yields

$$
P=\int_{-\infty}^{\infty} d \epsilon \exp \left(-(\epsilon+\exp (-\epsilon)) \Pi_{k \neq j} \exp \left(-\exp \left(\eta_{i k}-\eta_{i j}-\epsilon\right)\right) .\right.
$$

We make a change of variables by setting $t=\exp (-\epsilon)$, so $d \epsilon=-d t / t$. Evaluating the integral in the new variables yields

$$
\begin{aligned}
P & =\int_{0}^{\infty} d t \exp (-t) \Pi_{k \neq j} \exp \left(-t \exp \left(\eta_{i k}-\eta_{i j}\right)\right) \\
& =\int_{0}^{\infty} d t \exp \left(-t \sum_{k=0}^{J} \exp \left(\eta_{i k}-\eta_{i j}\right)\right) \\
& =\frac{1}{\sum_{k=0}^{J} \exp \left(\eta_{i k}-\eta_{i j}\right)} \\
& =\frac{\exp \left(\eta_{i j}\right)}{\sum_{k=0}^{J} \exp \left(\eta_{i k}\right)}
\end{aligned}
$$

as desired. 
Corollary 8 (Expected marital preferences by observed types). Suppose a man with observable type $i$ and (unobservable) specific identity $g$ derives utility $V_{i j g}^{m}=\eta_{i j}^{m}+\sigma \epsilon_{i j g}$ from being married to a woman of observable type $j$, independent of her specific identity. If $\sigma>0, \eta_{i j}^{m} \in \mathbf{R}$ and $\epsilon_{i j g}$ are as in Lemma $\mathbf{q}$, then the probability he prefers a woman of type $j$ to all other alternatives in $\{0,1, \ldots, J\}$ is given by (2).

Remark 3 (The Boltzmann / Gibbs distribution). The probabilities which appear in (2) and (41) take the form of the Boltzmann or Gibbs distributions from statistical physics, in which the deterministic component $\eta_{i j}^{m}$ of the utility derived plays the role of the energy associated with marital state $j$, while the strength $\sigma$ of the random component plays the role of the physical temperature. This connection is also discussed by Galichon and Salanié [14] [15].

\section{Appendix B. The Legendre Transform}

Here some well-known results pertaining to convexity and the Legendre transform are recalled. Let $F: \mathbf{R}^{n} \rightarrow \mathbf{R}$ be a twice continuously differentiable function; $F$ is convex if $\operatorname{Hess}(\mathrm{F}):=D^{2} F \geq 0$, and strictly convex if the line segment connecting any two points on the graph of $F$ lies above the graph. The Legendre transform or convex dual function to $F(p)$ is denoted $F^{*}()$ and defined pointwise by:

$$
F^{*}(q)=\sup _{p \in \mathbf{R}^{n}}\{q \cdot p-F(p)\} .
$$

Since the supremum of affine functions is convex, it is clear that $F^{*}(q)$ is a convex function. Additionally, the following duality result is true:

Lemma 9 (Legendre duality). Let $F \in C^{2}$ be strongly convex on $\mathbf{R}^{n}$, meaning $\operatorname{Hess}(F)>$ 0 . Then $F^{*}$ is also twice continuously differentiable. Further, if $q=D F(p)$, then $p=D F^{*}(q)$.

\section{REFERENCES}

[1] Gary Becker. A theory of marriage, Part I. Journal of Political Economy 81 (1973) 813-846.

[2] Steven Berry and Peter Reiss. Empirical models of entry and market structure, chapter for Volume III of the Handbook of Industrial Organization, edited by Mark Armstrong and Robert Porter. New York: North Holland, 2007.

[3] Loren Brandt, Aloysius Siow and Carl Vogel. Large shocks and small changes in the marriage market for famine born cohorts in China. University of Toronto Working Paper, 2008.

[4] Pierre-Andre Chiappori, Robert J. McCann and Lars Nesheim. Hedonic price equilibria, stable matching, and optimal transport: equivalence, topology, and uniqueness. Economic Theory 42 (2010) 317354.

[5] Pierre-Andre Chiappori, Bernard Selanié and Yoram Weiss. Assortative matching on the marriage market: A structural investigation. In process.

[6] Eugene Choo, Shannon Seitz and Aloysius Siow. Marriage matching, risk sharing, and spousal labour supplies. University of Toronto Working Paper, 2008.

[7] Eugene Choo and Aloysius Siow. Who marries whom and why. Journal of Political Economy 114 (2006) 175-201.

[8] John K. Dagsvik. Aggregation in matching markets. International Economic Review 41 (2000) $27-57$. 
[9] Colin Decker. When do Systematic Gains Uniquely Determine the Number of Marriages between Different Types in the Choo-Siow Marriage Matching Model? Sufficient Conditions for a Unique Equilibrium. University of Toronto MSc. Thesis, 2010.

[10] Ivar Ekeland. Existence, uniqueness and efficiency of equilibrium in hedonic markets with multidimensional types. Economic Theory 42 (2010) 275-315.

[11] Alessio Figalli and Young-Heon Kim and Robert J. McCann. When is multidimensional screening a convex program? Journal of Economic Theory 146 (2011) 454-478.

[12] Jeremy T. Fox. Estimating matching games with transfers. University of Chicago working paper, 2009.

[13] Alfred Galichon. Discussion of A. Siow's 'Testing Becker's theory of positive assortative matching'. Milton Friedman Institute, University of Chicago. February 28, 2009.

[14] Alfred Galichon and Bernard Salanié. Matching with trade-offs: Revealed preferences over competing characteristics. Working paper, 2009.

[15] Alfred Galichon and Bernard Salanié. Cupid's invisible hand: social surplus and identification in matching models. Working paper, 2011.

[16] Neil E. Gretsky, Joseph M. Ostroy and William R. Zame. The nonatomic assignment model. Economic Theory 2 (1992) 103-127.

[17] Roger A. Horn and Charles R. Johnson. Matrix Analysis. Cambridge: Cambridge University Press, 1985.

[18] Felix Kuber and Karl Schmedders. Competitive equilibria in semi-algebraic economies. Penn Institute for Economic Research working paper, 2007.

[19] Daniel McFadden. Conditional logit analysis of qualitative choice behavior, in Frontiers in Econometrics, edited by Paul Zarembka, New York: Academic Press, 1974.

[20] Robert Pollack. Two-sex population models and classical stable population theory. In Convergent Issues in Genetics and Demography, edited by Julian Adams et al., 317-33. New York, Oxford: Oxford University Press, 1990.

[21] John H. Pollard. Modelling the interaction between the sexes. Mathematical and Computer Modelling 26 (1997) 11-24.

[22] Aloysius Siow. Testing Becker's theory of positive assortative matching. Working paper 356, University of Toronto Department of Economics, 2009.

[23] Aloysius Siow. How does the marriage market clear? An empirical framework. Canadian Journal of Economics, 41 (2008) 1121-1155.

Department of Mathematics, University of Toronto, Toronto Ontario Canada M5S 2E4 Colin.decker@utoronto.ca Current address: Sunlife Financial, 150 King Street West Toronto Ontario Canada M5H 3T9 ATTN:TK11 Corporate Risk Management

Departments of Mathematics and Physics, Jadwin Hall, Princeton University, P.O. Box 708, Princeton, NJ 08542, USA lieb@math.princeton.edu

Corresponding author: Department of Mathematics, University of Toronto, Toronto Ontario Canada M5S 2E4 mCcann@math.toronto.edu

Department of Mathematics, University of Toronto, Toronto Ontario Canada M5S 2E4 stephens@math.toronto.edu. Current address: Department of Mathematics, University of Washington, Seattle Washington USA 98195-4350 Benstph@math.washington.edu 